\title{
A Hybrid Simulation Approach for Piezoelectric Vibration Energy Harvesting
}

\author{
Dominik Gedeon 1 , Simon T. Meyer ${ }^{1}$, Stefan J. Rupitsch ${ }^{1}$, Reinhard Lerch ${ }^{1}$ \\ ${ }^{1}$ Chair of Sensor Technology, University Erlangen-Nuremberg (FAU), Paul-Gordan-Str. 3/5, 91052 \\ Erlangen, Germany, \\ Dominik.Gedeon@fau.de
}

\begin{abstract}
We present a hybrid modeling method for the simulation of vibration Energy Harvester devices on a system level. A Finite Element solver for coupled problems (CFS++) is linked to a Simulink-based circuit simulator. The method addresses the need for optimization of the electromechanical device, the electronic energy extraction circuit as well as its control. By employing the Finite Element Method, precise modeling of piezoelectric harvesting devices can be accomplished. The investigation of nonlinear electronics (e.g., switched converters, diodes, etc.), however, demands for efficient transient computation. We resolve this discrepancy by using a modal truncation method for the Finite Element model. Through an automated procedure, simultaneous optimization of the system components is enabled.
\end{abstract}

Key words: Vibration Energy Harvesting, FE Simulation, Electronics Simulation, Power Electronics, Coupled Field Simulation

\section{Introduction}

A piezoelectric vibration Energy Harvesting $(\mathrm{EH})$ device consists of an electromechanical part (i.e., the harvester) and an appropriate energy extraction circuit [7]. While the optimization of the vibrating structure calls for a precise and flexible Finite Element modeling approach [6],[8],[9], electronic circuits other than a simple linear network require an efficient calculation method in the time-domain. As both parts of the device interact with each other, they must be simulated in a coupled model [1]. The representation of a harvester by a simple 1 DOF model is often considered, when electronic circuits are investigated [2],[5]. This allows important electromechanical properties to be included by an equivalent circuit model inside a circuit simulator. Nevertheless, it lacks the aforementioned precision and flexibility on the mechanical side.

In this contribution, we apply the Finite Element Method (FEM) for the modeling of an EH with piezoelectric coupling. To investigate power extraction methods such as SSHI [1] (synchronized switch harvesting on inductor), we link our FE model with a Simulink electronic circuit simulator.

The paper is organized as follows: At first we describe the FE model as a starting point for our reduction method. Secondly, the mathematical background of the modal reduction method is explained. In the following section, we describe the structure of the Simulink model and the automated computation procedure. In the results section, we validate the reduction technique against the full FE model. The suitability of the simulation tool is shown by an example circuit, that has been described in the literature [5].

\section{Finite Element Modeling}

The Finite Element Method is a mathematical approach for the computation of partial differential equations. By discretizing a model in the spatial domain, it yields a system of ordinary differential equations (ODEs). As an example, Fig. 1 shows a discretized model geometry of an $\mathrm{EH}$ device, that consists of an aluminum sheet substrate, a tip mass, and a piezoelectric composite patch (PI DuraAct P-876.A12) [4]. The structure is driven by an acceleration $a_{\mathrm{ax}}(t)$ in clamped-free condition. The dimensions of the $\mathrm{EH}$ are summarized in Tab. 1. 
Tab. 1: Dimensions of the piezoelectric harvester.

\begin{tabular}{|c|c|c|c|c|}
\hline & Length & Width & Thickness & $\begin{array}{c}\text { Free } \\
\text { length }\end{array}$ \\
\hline $\begin{array}{c}\text { Alu } \\
\text { sheet }\end{array}$ & 100 & 40 & 0.5 & 87.7 \\
\hline $\begin{array}{c}\text { Tip } \\
\text { Mass }\end{array}$ & 20 & 60 & 4.4 & - \\
\hline
\end{tabular}

Considering the piezoelectric effect in the PZT material, the FE Method yields a system of ODEs, given in Eq. (1).

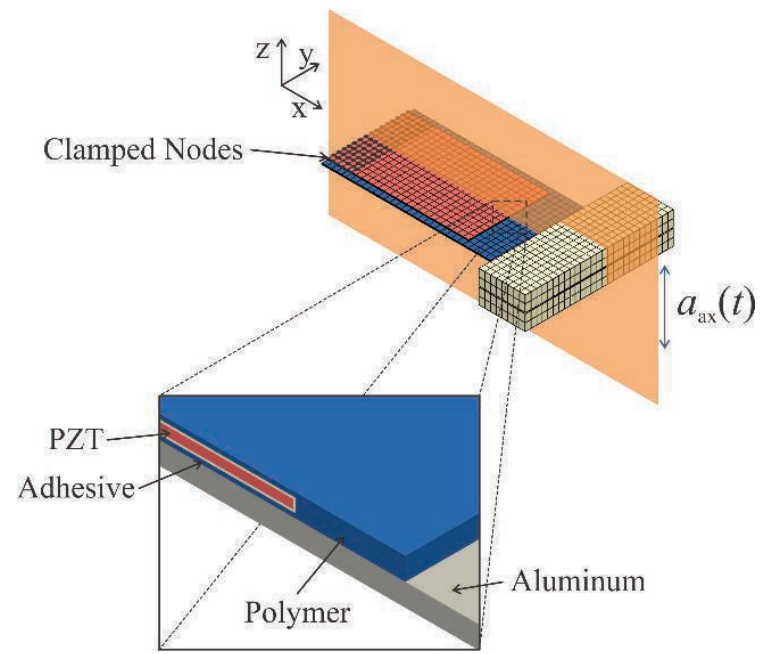

Fig. 1: FE model of a cantilever-type vibration energy harvester; magnification of cut through midsection plane.

$\left(\begin{array}{ll}0 & 0 \\ 0 & \mathrm{M}\end{array}\right)\left(\begin{array}{l}\ddot{\mathrm{V}} \\ \ddot{\mathrm{u}}\end{array}\right)+\left(\begin{array}{ll}0 & 0 \\ 0 & \mathrm{D}\end{array}\right)\left(\begin{array}{l}\dot{\mathrm{V}} \\ \dot{\mathrm{u}}\end{array}\right)+\left(\begin{array}{cc}-\mathrm{K}_{\mathrm{e}} & \mathrm{K}_{\mathrm{em}} \\ \mathrm{K}_{\mathrm{me}} & \mathrm{K}_{\mathrm{m}}\end{array}\right)\left(\begin{array}{l}\mathrm{V} \\ \mathrm{u}\end{array}\right)=$ $\left(\begin{array}{c}\mathrm{Q} \\ \mathrm{F}\end{array}\right)$.

In Eq. (1), M, D and $K_{m}$ are the mechanical massdamping- and stiffness matrices, $u$ is the vector of nodal displacements and $F$ stands for the vector of external nodal forces acting on the structure. $\mathrm{K}_{\mathrm{e}}$ denotes the electrostatic matrix, $\mathrm{V}$ is the vector of nodal potentials and $Q$ the vector of nodal electric charges. $\mathrm{K}_{\mathrm{em}}$ and $\mathrm{K}_{\mathrm{me}}$ are the coupling matrices, accounting for the piezoelectric effect. A dot above a symbol stands for differentiation with respect to time. Every nodal degree of freedom is represented by one equation. In the example considered here, Eq. (1) consists of almost 80.000 coupled equations, which constitute a remarkable computational effort for the time integration procedure.

\section{Modal Reduction}

From the theory of differential equations, it is known that the solution of a system of ODEs is a superposition of its weighted eigenfunctions.
The system (1) can therefore be transformed by means of its corresponding eigenvectors $\phi_{n}$. For the mechanical part of the system, these can be interpreted as mode shapes corresponding to resonant modes of the oscillating structure. They can be calculated numerically from the generalized eigenvalue (EV) problem

$\mathrm{K} \phi_{n}^{\mathrm{SC}}=\omega_{n}^{2} \mathrm{M} \phi_{n}^{\mathrm{SC}}$,

Where $\omega_{\mathrm{n}}$ is the corresponding eigenvalue. Considering only the first $n$ mechanical eigenmodes, the system is truncated to a much smaller set of ODEs by the relation (exemplified only for $\mathrm{M}$ ).

$\Phi_{\mathrm{SC}}^{\mathrm{T}} \mathrm{M} \Phi_{\mathrm{SC}}:=\mathrm{M}_{\mathrm{SC}}^{*}$.

Here, $\Phi_{\mathrm{SC}}$ is a matrix containing the first $n$ eigenvectors. A superscript $T$ stands for the transposed of a matrix.

$\Phi_{\mathrm{SC}}=\left(\phi_{1}^{\mathrm{SC}}, \ldots, \phi_{n}^{\mathrm{SC}}\right)$.

The electrostatic part can be reduced to capacitances between electrodes.

The system of equations, reduced by Eq. (3), however, produces valid results only for the case of electrical short-circuit. Calculating, for example, the electrical impedance of a piezoelectric structure produces erroneous results. The electric field, generated inside the piezoelectric material causes piezoelectric forces to act on the mechanical system. Consequently, the mode shapes used to reduce the system of equations are altered. For that reason, the reduced system is not fit to represent the general case of a piezoelectric device with arbitrary output voltages and electrical loads, like the full FE system in Eq. (1). This shortcoming can be overcome by solving a second eigenvalue problem, which incorporates piezoelectric coupling:

$\left(\begin{array}{cc}-\mathrm{K}_{\mathrm{e}} & \mathrm{K}_{\mathrm{em}} \\ \mathrm{K}_{\mathrm{me}} & \mathrm{K}_{\mathrm{m}}\end{array}\right) \phi_{n}^{\mathrm{OC}}=\omega_{n}^{2}\left(\begin{array}{cc}0 & 0 \\ 0 & \mathrm{M}\end{array}\right) \phi_{n}^{\mathrm{OC}}$.

The eigenvectors obtained by solving Eq. (5) correspond to the mode shapes in open-circuit condition. Analogously, a reduced set of ODEs is produced that is valid for the open-circuit case.

$\Phi_{\mathrm{OC}}^{\mathrm{T}} \mathrm{M} \Phi_{\mathrm{OC}}:=\mathrm{M}_{\mathrm{OC}}^{*}$.

The reduced set of equations in open-circuit condition accurately models the open-circuit voltage, whereas its counterpart in short-circuit condition is suitable for calculating the shortcircuit current. The internal impedance of an electric device is represented by a differential equation, relating open-circuit voltage to shortcircuit current. Hence, the internal impedance of the piezoelectric device can be modeled 
correctly by the aforementioned two sets of reduced ODEs.

Subsequently, the electrical properties of a piezoelectric harvesting device can be represented by its equivalent open-circuit voltage and its internal impedance $\underline{Z}_{\text {in }}(f)$.

Figures 2 and 3 show magnitude and phase angle of the internal impedance, respectively. The impedance was calculated from the full FE matrices and from the reduced model, considering five eigenmodes. The two models are in very good agreement up to the second harmonic of the harvester. It is clearly observable, that open-circuit resonance frequencies (corresponding to the minima in the magnitude curve) and the short-circuit resonance frequencies (corresponding to the maxima in the magnitude curve) coincide perfectly.

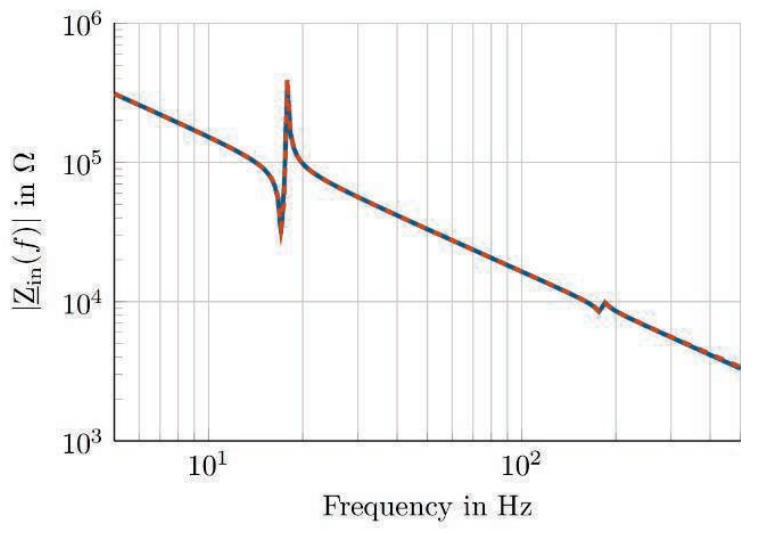

Fig. 2: Magnitude of internal impedance $\underline{Z}_{\text {in }}(f)$. Dashed line: full FE model; solid line: reduced model with five eigenmodes.

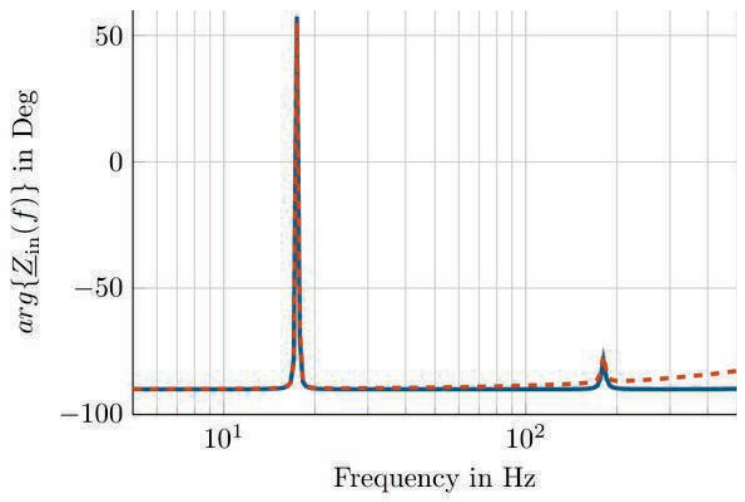

Fig. 3: Phase angle of internal impedance $\underline{Z}_{\text {in }}(f)$. Dashed line: Full FE model, solid line: Reduced model with five eigenmodes.

\section{Simulink Model}

The procedure of modal truncation and model setup can be easily automated in MATLAB/Simulink. The reduced FE model is represented by a subsystem with an electrical output port, which can be connected to a circuit. Simulink provides a suitable circuit simulation package (Simscape).

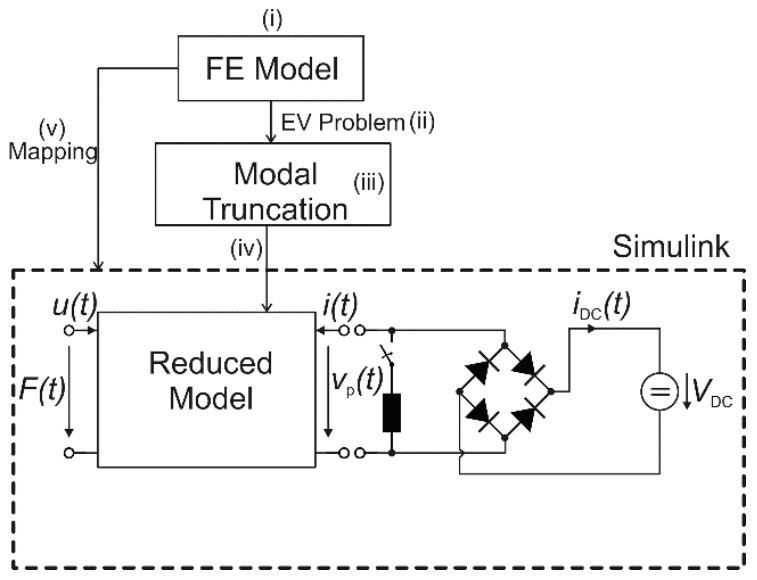

Fig. 4: Calculation procedure of model order reduction and automated buildup of hybrid model.

Figure 4 shows the schematic procedure of the model setup. Starting at a full FE model represented by a system of ODEs (i), the generalized EV problems are solved (ii). This computation is relatively cheap and yields the eigenvectors of the modeled geometry incorporating piezoelectric coupling. Subsequently, the original FE matrices are transformed into a modal subspace accounting only for the first $n$ eigenvalues (Modal Truncation) (iii) [3]. The truncated matrices are automatically included into a Simulink model (iv), where the resulting reduced set of ODEs can be solved by appropriate time-integration schemes. Relevant information from the model geometry (e.g., location of electrodes) are extracted and mapped to the Simulink model (v).

\section{Results}

To validate our simulation approach, we calculated output voltages $V_{\mathrm{R}}(f)$ of the full FE model in the harmonic domain. In doing so, an electric resistance served as electrical load. The same harmonic simulation was carried out with the reduced model. Furthermore, we evaluated the harvester's response in the time-domain with Simulink and performed a Fast-FourierTransform (FFT). The same was done with capacitive and inductive loads (not shown).

As can be seen from Fig. 5, the overall agreement of the two reduced calculation methods with the results from the full FE matrices is very good. 


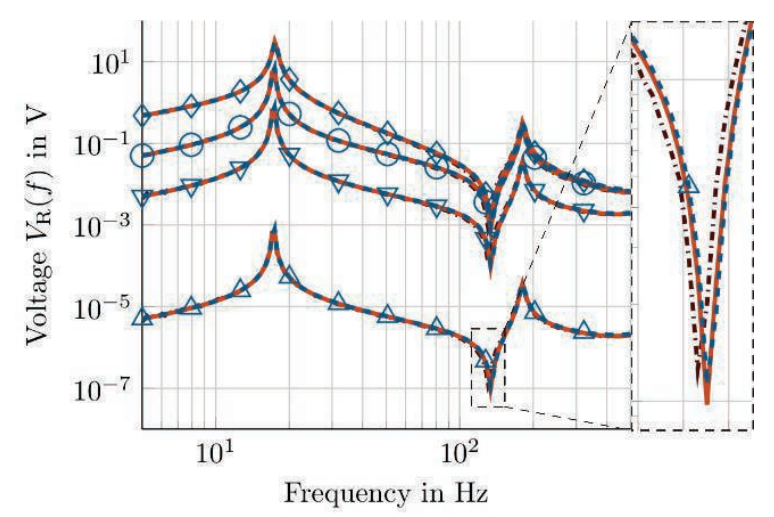

Fig 5: Parameter study of piezoelectric voltage at different resistive loads $R$. $\mathbf{\Delta}: R=1 \Omega ; \boldsymbol{\nabla}: R=1 \mathrm{k} \Omega ; \diamond$ : $R=10 \mathrm{k} \Omega$; $:: R=100 \mathrm{k} \Omega$. Dashed line: Full FE model; solid line: Reduced model harmonic calculation; dotdashed line: Reduced model Simulink calculation and subsequent FFT.

\section{Application Example}

As an application example, we investigated a technique known as SSHI (synchronized switch harvesting on inductor). The circuit is connected to the reduced model of the piezoelectric harvester (Fig. 4). It consists of three stages: A switched inductor (i), a diode rectifier (ii), and a DC-DC converter (iii), that regulates a constant DC voltage behind the rectifying bride. In our model, we neglected the DC-DC converter and replaced it by an ideal voltage source $V_{\mathrm{DC}}$. Figure 6 illustrates the principle of operation. The switch is turned into its conductive state, when the open-circuit voltage reaches a maximum or minimum. By connecting the piezoelectric device to an inductor, a resonant circuit is formed. After half the oscillation period, i.e, the zero-crossing of the current through the inductor, the switch is turned off. The output voltage of the harvester is thereby inverted. This causes the voltage to be stepped up during every half-period of the harvester's oscillation until it reaches the voltage at which the diodes become conductive. The oscillation in $i_{\mathrm{DC}}(t)$ is due to the second bending mode of the mechanical structure and would not be visible, if only one mode were considered in the model reduction.

The instantaneous power that is extracted from the vibrating harvester becomes

$p(t)=i_{\mathrm{DC}}(t) V_{\mathrm{DC}}$.

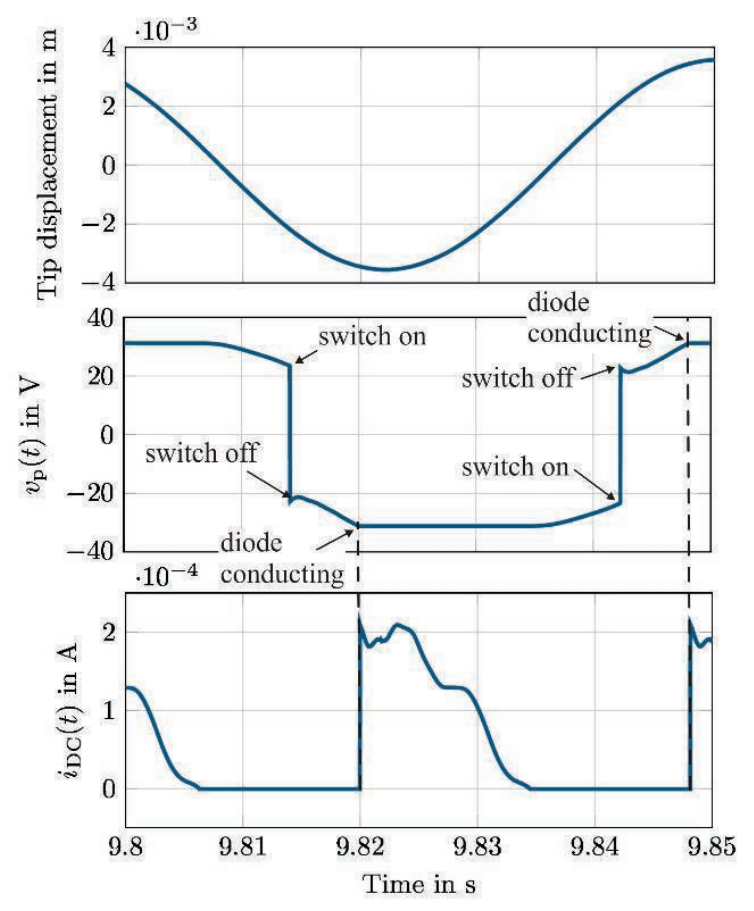

Fig. 6: Piezoelectric voltage $v_{p}(t)$ during two switching cycles; electric current behind the rectifier $i_{D C}(t)$ with $V_{D C}=30 \mathrm{~V}$; the excitation amplitude was $\hat{a}_{a x}=$ $1 \mathrm{~m} / \mathrm{s}^{2}$. The tip displacement represents the mechanical vibration of the harvester.

To investigate the effect of excitation frequency and $V_{\mathrm{DC}}$ on the electrical power that can be extracted, we performed parametric simulations with Simulink in the time-domain. At each value of $V_{\mathrm{DC}}$ and for each excitation frequency, ten seconds of time series data was acquired. The instantaneous power (7) was integrated and normalized by the duration. The result, shown in figures 7 and 8 , is the mean power with respect to excitation frequency and $V_{\mathrm{DC}}$.

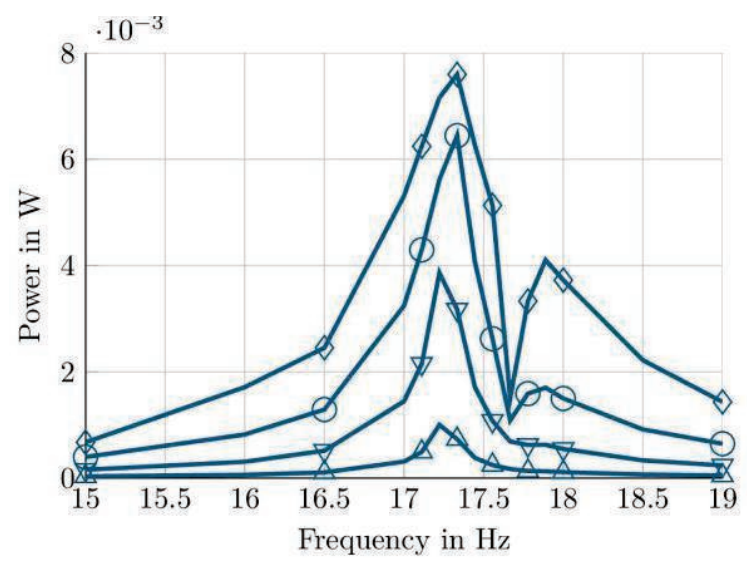

Fig. 7: Mean power harvested at different frequencies and different values of $V_{D C} . \mathbf{\Delta}: V_{D C}=1 \mathrm{~V} ; \boldsymbol{\nabla}: V_{D C}=$ $5 \mathrm{~V} ; \diamond: V_{D C}=15 \mathrm{~V} ; 0: V_{D C}=50 \mathrm{~V}$; the excitation amplitude was $\hat{a}_{a x}=1 \mathrm{~m} / \mathrm{s}^{2}$.

While the highest voltage amplitude in opencircuit condition can be observed at around $17.7 \mathrm{~Hz}$, the maximum mean power occurs below 
$17.5 \mathrm{~Hz}$. The drop in resonance frequency is caused by the electrical damping, introduced by the harvesting circuit.

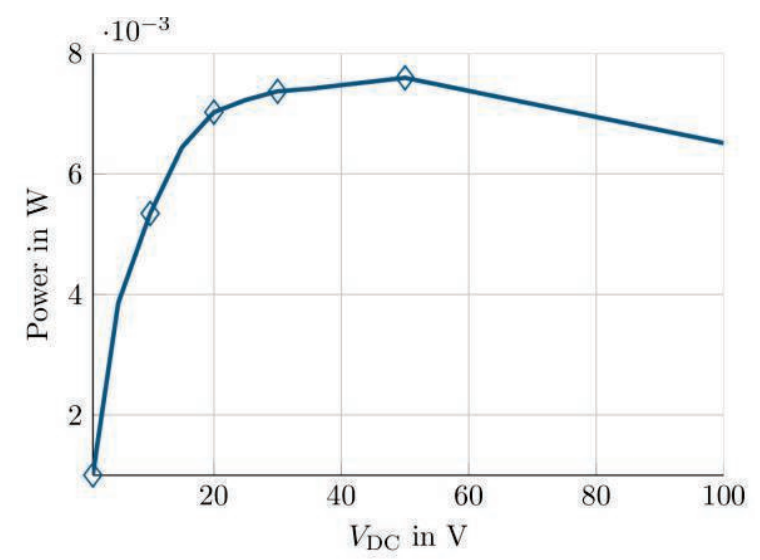

Fig. 8: Maximum mean power (from Fig. 7) over $V_{D C}$.

The maximum mean power also seems to be a function of the voltage behind the rectifier. In our example, a maximum mean power of $7.5 \mathrm{~mW}$ is harvested at $V_{\mathrm{DC}}=50 \mathrm{~V}$ and an excitation amplitude of $\hat{\mathrm{a}}_{\mathrm{ax}}=1 \mathrm{~m} / \mathrm{s}^{2}$.

\section{Conclusion}

We proposed an automated procedure for reducing the order of a FE model starting at the system of ODEs. While computational costs for the time integration are reduced by orders of magnitude, the resulting Simulink model is capable of accurately describing the dynamic electromechanical properties of the FE model. Therefore, one can couple such a model to a circuit simulator, which enables the simulation of nonlinear and time-varying circuits. We validated our approach by comparing the model output to the full FE system for a variety of electrical loads. Furthermore, we presented an example for a nonlinear switched circuit suitable for the extraction of energy from the electromechanical device.

\section{Acknowledgement}

This project was supported by German Research Foundation (DFG) as part of the special research field SFB/TR39. Furthermore, this contribution was supported by the Bavarian Ministry of Economic Affairs and Media, Energy and Technology as a part of the Bavarian project "Leistungszentrum Elektroniksysteme (LZE)".

\section{References}

[1] A. Daniels et. al., IEEE Trans Ultrason Ferroelectr Freq Control 60, 12 (2013), doi: 10.1109/TUFFC.2013.2861

[2] M. Deterre et al., Smart Mater. Struct. 21, 8 (2012), doi: 10.1088/0964-1726/21/8/085004
[3] N. G. Elvin, A. A. Elvin, J Intell SystStruct 20, 3-9 (2009); doi: 10.1177/1045389X08089957

[4] D. Gedeon et al., Ultrasonics Symposium (IUS), 2014 IEEE International, 2486-2489 (2014), doi: 10.1109/ULTSYM.2014.0620

[5] E. Lefeuvre, et al., J Electroceram 22, 171-179 (2009); doi: 10.1007/s10832-007-9361-6

[6] J. Park et. al., J. Mech. Schi. Technol. 26, 1 (2012), doi: 10.1007/s12206-011-0910-1

[7] H. A. Sodano et. al., J Intell Mater Sys Struct 16, 10 (2005), doi: 10.1177/1045389X05056681

[8] F. Wein et. al., Sruct Multidiscip O 48, 1 (2013)

[9] H. Wu et. al., J Intell Mater Sys Struct 24, 3 (2013), doi: 10.1177/1045389X12457254 